\title{
Las drogas sintéticas o nuevas sustancias psicoactivas, una amenaza latente sin controles efectivos*
}

\author{
Synthetic Drugs or New Psychoactive Substances, a Latent Threat without \\ Effective Controls
}

Javier Humberto Toro Gómez

Abogado, doctorando en Derecho de la Universidad de Baja California, Tepic, Nayarit,

México.jahutoro@hotmail.com

Fecha de recepción: 20 de julio de 2016

Fecha de aprobación: 9 de abril de 2017

Cómo citar este artículo/ to reference this article

Toro Gómez, J. (2017). Las drogas sintéticas o nuevas sustancias psicoactivas, una amenaza latente sin controles efectivos. Derecho Y Realidad, 15(29): e 8011. Recuperado de

https://revistas.uptc.edu.co/index.php/derecho_realidad/article/view/8011

Resumen: En este artículo se estudia de forma abreviada la amenaza mundial, latente y sin controles efectivos que representa la aparición de las drogas sintéticas o nuevas sustancias psicoactivas (NSP). La metodología empleada se basa en el enfoque cualitativo, utilizando herramientas como la revisión documental (informes oficiales, artículos periodísticos, de universidades y de organizaciones gubernamentales y no gubernamentales etc.). Inicialmente se describen aspectos básicos del contexto de las drogas de origen natural y sintéticas, luego se expone la caracterización del uso de drogas y el punto en el cual la sociedad actual y en especial los jóvenes y los niños presentan gran vulnerabilidad, y en último término, se propone una serie de estrategias con el fin de reducir el cosumo de NSP.

Palabras clave: droga sintética, drogas de origen natural, drogadicción, dosis personal, políticas públicas de control.

Abstract: This article presents in a synthetic way considerations about the global, latent and without effective controls threat that represents the arrival of synthetic drugs or new psychoactive substances (NSP, by its acronym in Spanish). This article is based on the qualitative approach, using tools such as document review (official reports, journalistic

\footnotetext{
* Artículo elaborado como complemento al tema de investigación de la tesis doctoral en Derecho. Universidad de Baja California (UBC), Tepic, México, abril de 2016.
} 
articles, from universities and governmental and non-governmental organizations, etc.), it starts by detailing basic aspects of the context of drugs of natural and synthetic origin, then focuses on the characterization of drug use, finding the point at which current society and especially young people and children are highly vulnerable, and finally it proposes a series of strategies in order to reduce the cost of NSP.

Keywords: synthetic drugs, drugs of natural origin, drug addiction, personal dose, public policies of control.

\section{Introducción}

La globalización ha tenido gran impacto en el mundo actual, no solo por la facilidad de comunicarse, sino además por las ventajas que ofrece el hecho de adquirir productos en un mercado aparentemente libre y sin restricciones. Pero ello conlleva peligros para los individuos, en especial para los jóvenes y los niños. Dentro de este fenómeno reciente se encuentra el consumo de drogas sintéticas como el éxtasis, LSD o anfetaminas, situación que de no ser enfrentada puede convertirse en un serio problema de salud pública.

La Comunidad Andina, con el auspicio de la Unión Europea, ha venido desarrollando un proyecto de cooperación denominado DROSICAN, que más allá de dar soluciones, pretende poner en marcha algunas iniciativas de prevención y poner en evidencia que países como Colombia, Bolivia, Perú y Ecuador han sido víctimas de la invasión de las drogas sintéticas. Además, este estudio señala que en Colombia, Perú y Ecuador el éxtasis es la sustancia más usada, mientras que en Bolivia lo son las anfetaminas, y también indica que adquirirlas no es difícil. Para los Estados miembros de la CAN, el problema de las drogas sintéticas es un fenómeno relativamente nuevo y que cobra cada vez mayor relevancia entre los jóvenes. Ya en el año 2006, en un primer estudio comparativo en los países andinos, realizado por la UNODC y CICAD, el éxtasis y los estimulantes anfetamínicos se encontraban entre las drogas de mayor consumo entre estudiantes de secundaria (DROSICAN, s.f.).

Así, la aparición de estas nuevas sustancias psicoactivas, denominadas "sintéticas", plantea grandes retos para las políticas de control de narcóticos de los países de América y en especial de América Latina, dada la inexistencia de políticas públicas referentes a esta situación, y la falta de controles de estas sustancias, ya que no lo establece ni el Convenio sobre Sustancias Psicotrópicas de 1991, ni la Convención Única de 1961 sobre Estupefacientes, pues sus componentes imitan fármacos o se derivan de sustancias que en el pasado estaban destinadas a rituales religiosos propios de los pueblos originarios de América.

En este orden de ideas, se puede pensar que los controles actuales son poco efectivos o ineficientes, por eso la intención de este documento es proponer herramientas para fortalecer el aparato estatal a partir de los instrumentos legales y constitucionales tanto de derecho interno como externo, para empezar a crear conciencia sobre el peligro y la amenaza de las drogas sintéticas no solo por sus efectos físicos y psicológicos, sino por lo que representan para el bien jurídico de la salud pública. 
Inicialmente, este artículo presenta una contextualización de las drogas de origen natural y las drogas sintéticas, y en este apartado menciona las referencias que han hecho mecanismos internacionales al respecto; después se muestra una descripción de las drogas sintéticas y, en último término, se proponen algunas estrategias para disminuir dicha amenaza y lograr controles efectivos en Colombia.

\section{Metodología}

Este artículo se desarrolla con base en el enfoque cualitativo, utilizando herramientas como la revisión documental (informes oficiales, artículos periodísticos, de universidades y de organizaciones gubernamentales y no gubernamentales etc.) y la dialéctica. El método empleado es de tipo descriptivo, por esta razón se inicia detallando aspectos básicos del contexto de las drogas de origen natural y sintéticas, y luego se enfoca en la caracterización del uso de drogas.

\section{Las drogas de origen natural y sintético: contexto}

Recientemente, la política internacional de lucha contra las drogas centró su atención en combatir las drogas denominadas de "origen natural"1, como la marihuana, la cocaína, el bazuco (base coca) y otras de origen vegetal con algún tipo de mezcla para su mejor oferta. Sin embargo, desde hace diez años aproximadamente, se ha empezado a hablar de "drogas sintéticas", es decir, aquellas que se elaboran en laboratorios y cuyos efectos en la salud física y metal de quienes las consumen, tienden a ser en su mayoría nefastos.

Hay que precisar que la definición de sintética o natural de una droga no tiene que ver con su mayor o menor toxicidad, ni con su mayor o menor poder adictivo, sino únicamente con su origen (Pons, 2016). Pero, desde luego, las drogas sintéticas, al ser artificiales y usar componentes de diferentes orígenes (por lo general más tóxicos), pueden generar efectos mayores.

En este punto es preciso traer a colación el estudio de consumo publicado por la Comunidad Andina de Naciones en el año 2012, en el que se señaló que el consumo de drogas sintéticas en Colombia, Bolivia, Perú y Ecuador, mostraba las siguientes variables:

Estimulantes tipo anfetaminas:

- La prevalencia del último año de consumo de ETA fue de 0,2\% entre los estudiantes de Bolivia, 0,9\% en Colombia, 0,7\% en Ecuador y 0,5\% en Perú.

- La percepción de gran riesgo frente al uso ocasional de éxtasis supera el $50 \%$ en Bolivia, llegando al $66 \%$ en Perú. Frente al uso frecuente de éxtasis, las cifras son aún mayores y varían desde el $75 \%$ en Bolivia hasta más de un $88 \%$ en Perú. En todos los países la percepción de gran riesgo para el uso de éxtasis es mayor entre las estudiantes mujeres. La prevalencia de último año de consumo de otras drogas que

\footnotetext{
${ }^{1}$ En realidad, las drogas llamadas naturales son semisintéticas en la gran mayoría de los casos, pues son muy escasas las preparaciones que se ingieren directamente del producto natural sin ser sometido este a algún procedimiento químico. En todo caso, las drogas sintéticas son elaboradas de manera totalmente artificial (Pons, 2016).
} 
resulta importante destacar, son las siguientes: $3,2 \%$ de LSD y $1,0 \%$ de hongos alucinógenos en Colombia. El hachís presenta registros de consumo reciente de 0,5\% en Colombia y 0,3\% en Ecuador y Perú. (Comunidad Andina, 2012a)

$\mathrm{El}$ aumento del consumo de drogas por parte de las mujeres, en relación con los hombres, es alarmante, y los efectos del consumo son sufridos por las familias y por la sociedad, más aun cuando se despiertan mayores comportamientos antisociales, tal como lo aseguran Muñoz, Graña, Peña y Andreu: "A diferencia de los varones, las mujeres que exhiben un comportamiento más antisocial realizan un consumo de estimulantes y drogas sintéticas significativamente superior que aquellas en las que este tipo de conductas es menos frecuente" (2002, p. 314).

De esos estudios, con muestras tan evidentes del consumo progresivo y acelerado de drogas sintéticas, nace el proyecto DROSICAN, cuyo propósito principal consistió en mostrar lo preocupante del uso y abuso de algunas sustancias específicas, entre ellas las drogas sintéticas, como también altos signos de dependencia. Otro hallazgo muy relevante en dicho estudio fue el importante uso de sustancias inhalables entre la población estudiantil y, en algunos países, con mayor incidencia en las mujeres.

Casi de manera inmediata, la situación anterior llamó la atención de los gobiernos y sus autoridades, pero desafortunadamente de manera tan leve y con tan bajo impacto, que ni siquiera pudieron sugerir una política pública de lucha contra las drogas, para empezar, asî fuera de manera incipiente, a combatir este nuevo fenómeno. Y a lo anterior hay que agregar la falta de consideración de la legalización de las drogas en escenarios nacionales y menos internacionales, como en el caso de Colombia, pues difícilmente se podrían asumir nuevas posturas frente al hecho de la comercialización de drogas sintéticas.

Pese a ello, la Comunidad Europea y la ONU, a través de informes y estudios, han manifestado su preocupación por el aumento no solo del consumo de las drogas sintéticas, sino de los adictos, el descontrol en mercados ilegales, las crecientes estrategias de venta a través de internet y obviamente el aumento de las muertes debido a los efectos de estas drogas, donde el principal problema es el desconocimiento de las sustancias consumidas y sus efectos.

En relación con dichos efectos, Villacís (2013) afirma que la preocupación más grande sobre las drogas sintéticas que más se consumen, como es el caso del éxtasis, es que la gente no sabe qué es lo que realmente está consumiendo. El doctor Cruz J (2013, pág. 43), afirma que "hay personas que se están pegando cualquier pepa creyendo que es éxtasis y pueden tener reacciones no previstas porque no saben qué se están tomando". Todo esto se refleja en que el éxtasis no necesariamente puede producir estimulación en el cerebro, sino que también puede provocar taquicardia o arritmia cardiaca.

Dentro de los efectos del éxtasis se puede mencionar que este puede hacer que quien lo consume se sienta más alerta y sintonizado con el ambiente, más feliz y tranquilo, con una sensación de cariño hacia otras personas. Los sonidos, colores y emociones son más intensos. Se percibe más energía, lo que permite bailar durante períodos más largos de tiempo, pero esa experiencia tiene graves efectos secundarios, por ejemplo, la temperatura corporal, la tensión arterial y el ritmo cardíaco aumentan.

Otros efectos físicos incluyen dolores musculares, náuseas, agarrotamiento de la mandíbula y rechinar de dientes. Se puede también experimentar sudor profuso, temblores y palpitaciones, sentirse deshidratado, confuso y cansado. Pero ¿cuáles son los verdaderos riesgos? La mayoría de las drogas, cuando son legales, se prueban primero en animales, pero en el caso 
de los consumidores de éxtasis y de la mayoría de las drogas sintéticas, ellos son los conejillos de indias.

Las investigaciones muestran que los consumidores habituales de fin de semana experimentan una "crisis" a mitad de semana, que los deja muy cansados y deprimidos, a menudo durante días, por ello a largo plazo se puede correr el riesgo de desarrollar problemas o incluso la muerte, debido a insolaciones, infartos o crisis de asma (Armenta \& Jelsma, 2015).

El panorama no es muy alentador, y tal conclusión se respalda en el último informe de la ONUDC, Oficina de las Naciones Unidas contra la Droga y el Delito, reportado en el año 2015, dado que la situación de salud, el tráfico de estupefacientes y el consumo no han presentado cambios significativos. Refiere el estudio que en la lista de estupefacientes sometida a fiscalización internacional (Oficina de Naciones Unidad contra las Droga y el Delito, s.f.), lista amarilla de 1971, se enumeró un promedio de 400 sustancias controladas y propulsores químicos, con un alto riesgo para la salud humana.

Pero, para la fecha, la lista amarilla es incierta, por las variadas mutaciones y aleaciones químicas creadas entre fármacos depresivos, estimulantes sexuales, fármacos utilizados en medicina veterinaria y psiquiátrica, además de la efedrina y pseudoefedrina, (materia prima de las anfetamina), situaciones que llevan a cuestionarse si existe un control por parte del Estado y sus autoridades frente a la comercialización, la distribución y el consumo de este tipo de sustancias, así como en la identificación de organizaciones delincuenciales dedicadas a dicha actividad criminal.

La preocupación de la Comunidad Europea no es vana, pues la Evaluación Global de las Drogas Sintéticas muestra el consumo de estimulantes de tipo anfetamínicos y nuevas sustancias psicoactivas (Oficina de Naciones Unidad contra las Droga y el Delito, 2014). Las encuestas del Eurobarómetro realizadas entre 12.000 jóvenes de 15 a 24 años de edad en toda la UE en el 2011, revelaron que la prevalencia de vida de los jóvenes que habían experimentado con las "sustancias legales que imitan los efectos de las drogas ilícitas" presentaron resultados que no están lejos de las tasas de uso de algunas sustancias sometidas a fiscalización.

Además revelaron que el uso de las NSP en la UE parece ser un poco más bajo que las tasas de consumo de anfetamina, pero similar a las tasas de uso de "éxtasis" y mayor que las tasas de uso de cocaína, LSD o heroína; que las tasas de uso de NSP más altas fueron reportadas por Irlanda con $16,3 \%$, seguido por Polonia con $9 \%$, Letonia con $8,8 \%$ y el Reino Unido con $8,2 \%$, y finalmente que los visitantes de clubes nocturnos muestran altas tasas de uso (Oficina de Naciones Unidad contra las Droga y el Delito, 2014).

\section{Las nuevas drogas sintéticas}

En ese contexto, las drogas sintéticas, o nuevas sustancias psicoactivas (NSP), son hoy en día un grave problema para el Estado, en un contexto mundial, y, por lo tanto, para los organismos de la salud y lógicamente para las autoridades judiciales, en razón a que su procesamiento es de fácil disposición, como quiera que, en primer lugar, pueden ser elaboradas cómodamente en el lugar de residencia y no exigen la utilización de laboratorios ni equipos sofisticados para su procesamiento.

En segundo lugar, la comercialización de las drogas sintéticas no es compleja, porque las organizaciones criminales dedicadas a esta actividad utilizan principalmente las redes 
sociales para acaparar toda la información, propagar publicidad de sus productos y organizar eventos - generalmente fiestas electrónicas clandestinas-, en donde se encargan de distribuir de forma descarada y ágil todo tipo de sustancias, las cuales, para empeorar la situación, son de fácil ocultamiento, pues se camuflan en aerosoles, bebidas, pastillas, aceites, entre otras múltiples presentaciones que son de difícil rastreo. Además, los precios de venta oscilan según el nivel social de los compradores y los efectos que producen, y hay que resaltar que la mayoría de los compradores son niños y jóvenes.

Los estudios sobre el consumo de drogas sintéticas en adolescentes son alarmantes, según se desprende de lo reportado por Sánchez y Suelves:

Un $51,4 \%$ y un $41,6 \%$ de los sujetos, respectivamente, declaró no haber consumido nunca tabaco ni alcohol, aunque se halló un $4,8 \%$ de fumadores diarios. Muy pocos sujetos declararon haber consumido las demás sustancias (un 7,1\% había consumido alguna vez cannabis y un 1,4\% drogas sintéticas). (Sánchez \& Suelves, 2001, pág.15) (negrillas fuera de texto)

Hay que anotar que la preocupación internacional no paró allí, el crecimiento desmesurado y sin control de las drogas sintéticas hizo que la Asamblea General de las Naciones Unidades estudiara la sugerencia hecha por el Gobierno colombiano en el año 2012, donde se deprecó el adelantamiento de las sesiones programadas para el año 2018 y 2019, para el año 2016, propuesta que fue acogida por los gobiernos de México y Guatemala, llevándose a cabo una sesión especial el 21 de abril del año 2016.

En dicho escenario, la Asamblea General, dentro del programa de las sesiones extraordinarias, "[...] examinó los progresos realizados en la aplicación de la Declaración Política y el Plan de Acción sobre Cooperación Internacional en Favor de una Estrategia Integral y Equilibrada para Contrarrestar el Problema Mundial de las Drogas [...]" (Oficina de Naciones Unidas contra las Droga y el Delito, 2015), y además replanteó la política mundial sobres las drogas y la determinación del uso de sustancias psicotrópicas para fines médicos.

Lo anterior causó el efecto contrario, dado que al ser de fácil adquisición por cualquier persona y por la dificultad de su control por parte de las autoridades, se agudizó la problemática. En conclusión, los Estados miembros decretaron un nuevo enfoque en la política antidroga, centrado en la persona, la salud pública y los derechos humanos, pero sin incluir controles materialmente efectivos sobre la situación.

Entre las drogas sintéticas más populares y conocidas está el éxtasis - técnicamente llamado MDMA y conocido popularmente como Eva, Adán, XTC, mitsubishis, supermanes-, y el LSD, Two CB (2 CB), que contiene diletamida de ácido lisérgico. Casi de manera estandarizada se presentan en pastillas con logos en papel y cartón para ser colocadas debajo de la lengua. 


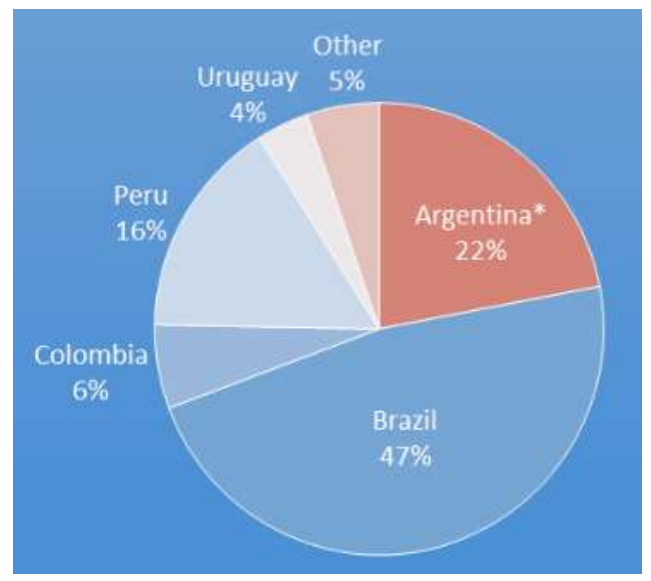

Figura 1. Éxtasis en América del Sur, América Central y el Caribe.

Fuente: Oficina de Naciones Unidad contra la Droga y el Delito (2014).

También ha aparecido una nueva droga, conocida como droga "zombie", al parecer descubierta en Brasil y Argentina, y ya de uso común entre universitarios y población juvenil en edad escolar. Lo que se desconoce en la actualidad es el número de sustancias químicas procesadas en laboratorios ilegales que no tienen control por parte de organismos de salud ni de las autoridades judiciales.

Dentro de estos narcóticos que no han tenido control se destacan estimulantes anfetamínicos y nuevas sustancias psicoactivas (NSP), entre otras nuevas modalidades de drogas sintéticas, coligiéndose de todo ello que la amenaza crece sin control, pues cada día se descubren nuevos escenarios y campos de venta, siendo la población infantil la más vulnerable, por el fácil acceso a redes de comunicación virtual.

Surge entonces la pregunta ¿se ha perdido la lucha contra las drogas? Y, precisamente, ese es el interrogante que permanentemente se plantea la Organización de las Naciones Unidas, pues las políticas diseñadas para combatir la problemática de drogadicción hasta hoy han sido insignificantes, comparadas obviamente con el crecimiento acelerado y sin control del flagelo. En efecto, está demostrado que la elaboración de planes de acción, los convenios para la cooperación, las leyes y los decretos internos por parte de los Estados han fracasado. A las sustancias de estupefacientes procesadas a partir de opiáceos, al cannabis y la cocaína se suman las drogas sintéticas como estimulantes de tipo anfetamínico y nuevas sustancias psicoactivas, las cuales se constituyen en armas letales para los jóvenes, como principales consumidores. Además se evidencia que el consumo de mefedrona, ketamina, cannabinoides sintéticos y sustancias inyectables, produce una mayor adición (Armenta \& Jelsma, 2015).

No obstante, el panorama no es del todo desalentador, pues hay que indicar que las Naciones Unidas, a través de la Junta Internacional de Fiscalización de Estupefacientes de la Oficina contra las Drogas y el Delito, efectuó un diagnóstico de la situación mundial que se está viviendo, lo que generó una serie de recomendaciones, tomadas frente a las cifras actuales de tráfico y consumo por regiones, y demostró que se han elaborado controles y políticas para mitigar este infortunio, siendo la unidad de todos los Estados miembros lo que puede permitir contrarrestar las nuevas prácticas delictivas.

Así, según la Junta Internacional de Fiscalización de Estupefacientes (JIFE) de la Oficina de Naciones Unidad contra la Droga y el Delito (ONUDC): 
[...] el proceso de conectar lo local con lo mundial, se está llevando a cabo actualmente, intercambiando las mejores prácticas y enseñanzas extraídas de las experiencias locales integradas en las estrategias nacionales. Ello refuerza la labor de la UNODC, la Comisión de Estupefacientes y otras entidades para seguir fomentando la cooperación Sur-Sur (un amplio marco de colaboración entre los países del Sur), de conformidad con los Principios Rectores de las Naciones Unidas sobre el Desarrollo Alternativo. (Oficina de Naciones Unidad contra la Droga y el Delito, 2015)

A la misma conclusión llegó el referido informe sobre evaluación mundial de las drogas sintéticas de 2014, en el cual se dejó establecido lo siguiente:

En primer término, la expansión mundial del mercado de estimulantes de tipo anfetamínico y la naturaleza dinámica de la situación de las NSP realzan la necesidad de una vigilancia global y de un intercambio de conocimientos a partir de las experiencias individuales de los distintos Estados Miembros. El examen de la evidencia científica, datos forenses y de la información son esenciales para generar una respuesta eficaz al rápido crecimiento del mercado de drogas sintéticas. A efectos de complementar el mecanismo actualmente disponible para la vigilancia mundial de las drogas sintéticas, tal como el Programa Mundial de Vigilancia de las Drogas Sintéticas: Análisis, Informes y Tendencias (SMART), UNODC puso en marcha el Sistema de Alerta Temprana (EWA) sobre las NSP en junio de 2013. El Sistema de Alerta Temprana está diseñado para permitir el intercambio oportuno y completo de información sobre las NSP, incluyendo metodologías analíticas, documentos de referencia y datos de análisis de tendencias, con el fin de proporcionar un punto de referencia mundial. (Oficina de Naciones Unidad contra las Droga y el Delito, 2014)

En resumen, es urgente implementar y fortalecer los mecanismos creados por la Oficina contra las Drogas y el Delito, y celebrar las reuniones extraordinarias que tienen como fin tomar las medidas pertinentes para la disminución del consumo, los riesgos y la distribución, dado que, por lo menos en el caso de Colombia, un país que, pese a su reconocida y loable lucha frontal contra las drogas, no ha logrado su erradicación definitiva.

Otro propósito de las reuniones es llevar a cabo campañas de prevención e información sobre las nuevas sustancias psicoactivas, de la mano con las políticas públicas de cooperación entre Estados y de entidades gubernamentales, con el fin único de poder superar, en un futuro no muy lejano, la problemática que se ha venido viviendo, pues, de lo contrario, se seguirá perdiendo la batalla, como ha sucedido con las sustancias derivadas del opio, cannabis y cocaína, lo que sin lugar a dudas en otra década nos tendrá todavía haciendo estudios sin resultados en la vida práctica.

\section{Estrategias para disminuir la amenaza de las drogas sintéticas y lograr controles efectivos en Colombia}

Colombia es un país que se ha visto azotado por el flagelo de las drogas de manera contundente y reiterada. En los últimos 50 años ha dado una lucha incansable para tratar de erradicar el cultivo masivo y el microtráfico de estupefacientes, ha legislado y desarrollado jurisprudencia alrededor del tema de manera profusa, pero desafortunadamente aún quedan sin legislar conductas irregulares y lesivas de bienes jurídicos, que ni siquiera han sido elevadas a la categoría de delito, como ocurre con el caso de las drogas sintéticas. 
Lo anterior está respaldado en el hecho de que solo se haya proferido la primera condena a pena privativa de la libertad por un delito de esta envergadura hasta el año 2015, es decir, casi tres décadas después de estarse comercializando alucinógenos producidos en laboratorio, como el éxtasis o el LSD. Por si fuera poco, se estima que las normas sobre estupefacientes que están vigentes en Colombia, tienen más de 30 años de retraso y ya hay más de cien mil colombianos que han consumido alguna vez estas sustancias.

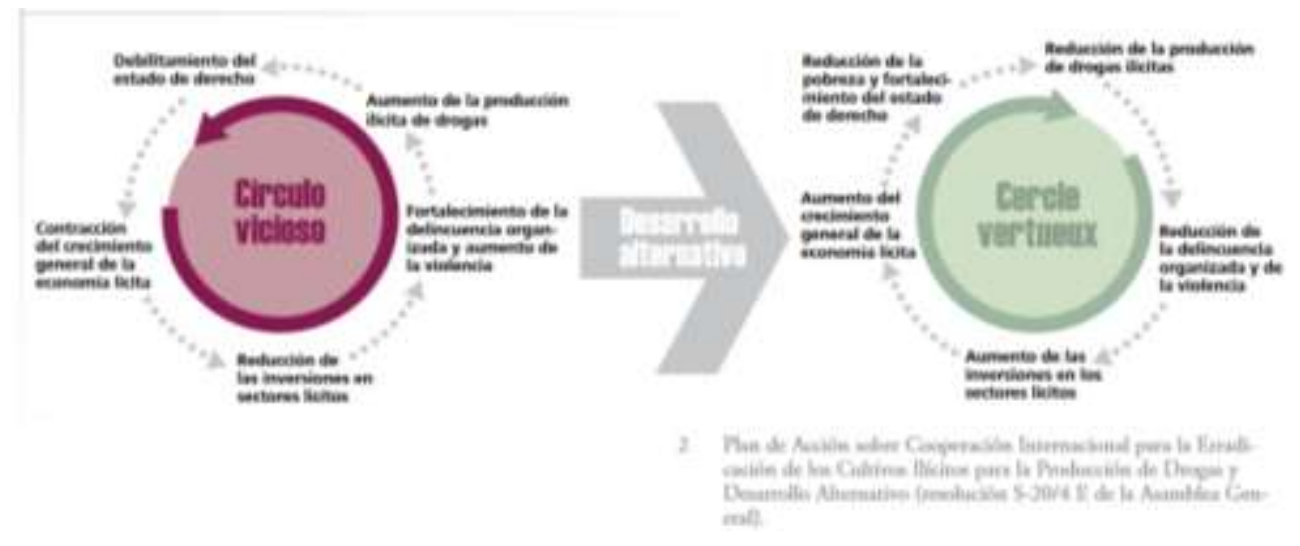

Figura 2. Repercusiones de la producción de drogas ilícitas y de las intervenciones de desarrollo alternativo.

Fuente: Oficina de Naciones Unidad contra la Droga y el Delito (UNODC, informe mundial sobre las drogas 2015).

La Figura 2 representa la causa y los efectos del debilitamiento del Estado de derecho y su relación con las drogas, de ahí que si se fortalecen las instituciones estatales y el derecho, se efectuará un cambio en el flagelo de las drogas que ha afectado a todo el mundo.

Tomando todo ello en consideración, las posibilidades o alternativas que este artículo plantea, son las siguientes:

i) Endurecimiento de penas y sanciones, reforma a la justicia penal. Aunque la respuesta estatal, por regla general, es el endurecimiento de las penas, ello no siempre significa que sobre quien recae el peso de la ley tema a las consecuencias de sus actos. No obstante, de alguna manera la legislación debe adaptarse a los cambios que el mundo actual le revela. Las cifras, por lo menos en el caso de Colombia, demuestran las dificultades que enfrentan Policía y Fiscalía para sacar de la calle a las redes de productores y distribuidores de ese tipo de drogas, pues aunque se realizan capturas, ninguna persona llega a los jueces, tal y como lo ilustra la prensa escrita, cuando ponen en evidencia casos como este: a pesar de haber sido capturado en flagrancia en Bogotá a finales del año pasado por transportar dos mil millones de pesos en drogas sintéticas en un maletín, un hombre de 34 años estuvo apenas unas horas ante un juez y al final recuperó su libertad. La Policía lo capturó llevando cuatro tiras de papel (trips) impregnadas con LSD, uno de los ácidos más temidos del mundo de las drogas. Pero no pudieron avanzar en su caso porque el peso de las hojas apenas era de cuatro 
gramos y no hubo manera de establecer, con pruebas de laboratorio, cuál era el tipo de droga transportada (El Tiempo, 2016).

Es necesaria entonces una revolución legislativa al respecto, que permita dejar de manejar el asunto "a ojo" o vía interpretación de la ley, porque, a diferencia de otras sustancias como la cocaína, la marihuana o el hachís, en el caso de las drogas sintéticas no hay dosis mínima establecida y rara vez se puede diferenciar entre un consumidor y un distribuidor.

ii) Actualización de químicos y compuestos, para prohibir su comercialización. Ya se dijo que las herramientas legales actuales son insuficientes para poder establecer a través de pruebas de laboratorio el tipo de droga de que se trata, lo que obliga a que los Estados pongan en marcha novedosas estrategias de control, más allá de la discusión semántica entre "combatir y legalizar". Para ello deberán entonces preguntarse ¿Qué van a hacer los países cuando sus hijos adquieran estos "nuevos compuestos", que debido a la tecnología sofisticada tendrán modificaciones y "mejoras" incesantes, que dificultarán su determinación legal como una droga ilícita y circularán sin problemas por vacíos legales, como sustancias "energizantes o recreativas"? ¿Qué van a hacer los países si a pesar de establecer que se trata de compuestos prohibidos, los mismos se mezclan con otros de libre circulación o de circulación legal y se siguen camuflando en llamativas pastillas rosas o en papel cartón parecido a los stickers de cuaderno? Y el problema es aún mayor porque mientras se incluye la droga o sustancia en la lista de Naciones Unidas como nociva, ya la misma ni siquiera existe en el mercado, pues al año es posible que surjan más de 200 drogas nuevas, producto de la variación de las moléculas en sofisticados laboratorios criminales (El Tiempo, 2016).

iii) Fortalecimiento de unidades de persecución de este tipo de delitos. Colombia marca la pauta en la identificación de las más recursivas modalidades para el tráfico de drogas denominadas tradicionales - cocaína, heroína, marihuana-, pero en el enfrentamiento de las llamadas "drogas sintéticas", la situación no es pacífica y para la muestra, declaraciones como las que dio en un artículo web el coronel Miguel Antonio Tunjano, jefe del Centro Internacional de Estudios Estratégicos de la Policía Antinarcóticos, cuando aseguró: "Nuestros policías se guían por el color de la pastilla, por ejemplo: si es rosada, inmediatamente la etiquetan como $2 \mathrm{CB}$. Pero la verdad es que sin enviarla a un laboratorio no podemos establecer cuál es su molécula química" (El Tiempo, 2016).

La otra gran preocupación de las autoridades es que mientras que los traficantes siguen incrementando sus ganancias y extendiendo su presencia no solo en las grandes ciudades, sino en las regiones, también van en aumento los casos de afectados por falsificación y adulteración de ese tipo de sustancias, a veces con componentes mortales.

iv) Políticas públicas de educación. Este tema no es novedoso, lo que ocurre es que en el caso de Colombia, ningún gobierno se ha comprometido con el tema educativo, pues están más preocupados por la burocracia y lo que se necesita es voluntad política, dar el salto de ministros y policías preocupados a acciones contundentes, políticas públicas reales que eviten el crecimiento y desbordamiento del flagelo. 
Bien lo dijo el honorable magistrado de nuestra llamada "Corte Constitucional de Oro" el doctor Carlos Gaviria Díaz, en la sentencia C 221 de 1994,

\begin{abstract}
¿Qué puede hacer el Estado, si encuentra indeseable el consumo de narcóticos y estupefacientes y juzga deseable evitarlo, sin vulnerar la libertad de las personas? Cree la Corte que la única vía adecuada y compatible con los principios que el propio Estado se ha comprometido a respetar y a promover, consiste en brindar al conglomerado que constituye su pueblo, las posibilidades de educarse. ¿Conduce dicha vía a la finalidad indicada? No necesariamente, ni es de eso de lo que se trata en primer término. Se trata de que cada persona elija su forma de vida responsablemente, y para lograr ese objetivo, es preciso remover el obstáculo mayor y definitivo: la ignorancia. No puede, pues, un Estado respetuoso de la dignidad humana, de la autonomía personal y el libre desarrollo de la personalidad, escamotear su obligación irrenunciable de educar, y sustituir a ella la represión como forma de controlar el consumo de sustancias que se juzgan nocivas para la persona individualmente considerada y, eventualmente, para la comunidad a la que necesariamente se halla integrada. (Corte Constitucional, sentencia C-221, 1994).
\end{abstract}

Específicamente, la educación para prevenir que los niños y adolescentes entren en la drogadicción es un trabajo cooperativo del Estado, las instituciones educativas y la familia, por eso a cada edad se le debe hacer el refuerzo de algunas temáticas, así:

A partir de los 6 años y hasta los 12 hay que seguir trabajando todos estos valores y, además, actitudes hacia la salud, autoestima y habilidades de interacción. A partir de los 12, es necesario trabajar específicamente la toma de decisiones, las habilidades de oposición y las actitudes hacia las drogas. (abc.es, s.f.) (Subrayado fuera de texto)

Reflexión profunda que nos invita a pensar en la importancia de la educación para cualquier ciudadano del mundo, pero no la educación a través de herramientas como el castigo y la represión, sino a través de la prevención y la concienciación de valores como la vida, el autorrespeto, la integridad física, la salud física y mental, entre otros.

v) Uso adecuado de plataformas y redes sociales. Por ser el medio más utilizado para la comercialización, la divulgación y el conocimiento de las sustancias que día a día aparecen en rumbas, eventos sociales y demás actividades, se hace necesario aprovechar este sistema de comunicación con el fin de entregarle a la población herramientas y alertas sobre los riesgos del consumo de sustancias sintéticas. También debe hacerse divulgación amplia en medios radiales y televisivos, que adviertan a la población vulnerable sobre las consecuencias de involucrarse en el consumo de sustancias psicotrópicas y químicas como las indicadas anteriormente.

El uso de las redes sociales es una excelente herramienta para advertir a las personas sobre los efectos de las drogas sintéticas, más aun cuando cada vez es más utilizado internet. En Colombia, por ejemplo, el "64\% de los colombianos ya usa internet", y quienes más lo 
utilizan son las personas que tienen entre 21 y 35 años (Ministerio de Tecnologías de la Información y las Comunicaciones, citado por El Colombiano, 2017), lo cual coincide con las edades propicias para educar sobre el consumo de drogas.

\section{Conclusiones}

La discusión sobre la receta correcta para combatir el problema de las drogas sintéticas parece no tener fin, y aunque en un momento dado parezca que se van a lograr consensos, ninguna de las posturas principales, el combate y la legalización, logra tener la suficiente contundencia para llegar a una definición, y a veces hasta se puede pensar que las dos tienen razones válidas y suficientes que justifiquen inclinarse por cualquiera.

La tendencia de los últimos años en los gobiernos latinoamericanos es enfrentar el problema del consumo de drogas con la legalización de sustancias psicotrópicas; por ejemplo, en Colombia ya se legalizó el consumo de la dosis mínima y el uso de drogas en tratamientos médicos, basados en estudios científicos sobre su uso medicinal.

El riesgo ocurre entonces con las drogas sintéticas o químicas, las cuales son aleaciones de diferentes sustancias farmacéuticas o caseras que aumentan el riesgo en la adición y en los daños en la salud humana.

Las drogas sintéticas son una amenaza latente, más aun por su variedad en componentes que generan daños irreparables principalmente a la población juvenil, que requiere una protección radical por parte de cada uno de los gobiernos, no solo de Latinoamérica sino también de todo el mundo, pues se tiene conocimiento que algunas de estas sustancias provienen de Europa, Asia y Estados Unidos. En otras palabras, es un problema global que requiere intervención local de manera inmediata.

Las herramientas que puede usar el Gobierno colombiano para tratar el flagelo de las drogas, son: 1. Endurecimiento de penas y sanciones, reforma a la justicia penal, 2. Actualización de químicos y compuestos, para prohibir su comercialización, 3. Fortalecimiento de unidades de persecución de este tipo de delitos, 4. Políticas públicas de educación, y 5. Uso adecuado de plataformas y redes sociales. 


\section{Referencias}

abc.es (s.f.). El rincón de la prevención. abc.es. Recuperado de http://www.abc.es/especiales/rincon-prevencion/educacion-en-valores-clave-paraprevenir-los-consumos-de-drogas/.

Armenta, A. \& Jelsma, M. (2015). Guía básica: las convenciones de droga de la ONU. Recuperado de https://www.tni.org/es/publicacion/las-convenciones-de-drogas-de-laonu.

Comunidad Andina. (2012a). II Estudio epidemiológico andino sobre consumo de drogas en la población universitaria, Informe Regional 2012. (1. ${ }^{\mathrm{a}}$ ed.). Recuperado de http://www.comunidadandina.org/StaticFiles/20132718338Informe_Regional.pdf.

Comunidad Andina. (2012b). II Estudio epidemiológico andino sobre consumo de drogas en la población universitaria, Informe Colombia 2012. (1. ${ }^{\mathrm{a}}$ ed.). Recuperado de http://www.odc.gov.co/Portals/1/publicaciones/pdf/destacados/CO03542012-iiestudio-epidemiologico-andino-sobre-consumo-drogas-poblacion-universitariainforme-colombia-2012-.pdf.

Congreso de la República de Colombia. (1986). Ley 30 de 1986, por la cual se adopta el Estatuto Nacional de Estupefacientes y se dictan otras disposiciones. Bogotá, DC: Imprenta Nacional.

Corte Constitucional de Colombia. (1994, 5 de mayo). Sentencia C-221 de 1994. Expediente n. ${ }^{\circ}$ D- 429. M.P. Carlos Gaviria Díaz.

DROSICAN. (s.f.). Drogas sintéticas una amenaza con respuesta comunitaria. Recuperado de http://www.comunidadandina.org/DS/DROSICAN/Brochure\%20DROSICAN.pdf.

El Colombiano. (2017, 1 de oct.). ¿Qué tanto tiempo gastan al mes los colombianos en redes sociales? $\quad$ El Colombiano. $\quad$ Recuperado de http://www.elcolombiano.com/tecnologia/tiempo-de-uso-de-redes-sociales-encolombia-HC7411548.

El Tiempo. (2016, 30 de abril). Las drogas sintéticas avanzan y el país no tiene leyes para atacarlo. El Tiempo. Archivo. Recuperado de http://www.eltiempo.com/archivo/documento/CMS-16578796.

Muñoz, M. J., Graña, J.L., Peña, M.E. \& Andreu, J.M. (2002). Influencia de la conducta antisocial en el consumo de drogas ilegales en población adolescente. Adicciones, 14(3), 313-320. Recuperado de http://adicciones.es/index.php/adicciones/article/view/486/481

Oficina de Naciones Unidad contra las Droga y el Delito. (2014). Evaluación global de las drogas sintéticas, 2014 Estimulantes de tipo anfetamínico y nuevas sustancias psicoactivas. 
https://www.unodc.org/documents/peruandecuador/Publicaciones/Publicaciones2014/ sustancias/Global_Synthetics_Drugs_Assessment_presentation_ES_Final.pdf.

Oficina de Naciones Unidad contra las Droga y el Delito. (2015). Informe mundial sobre las drogas, 2015 Resumen Ejecutivo. Recuperado de https://www.unodc.org/documents/wdr2015/WDR15_ExSum_S.pdf.

Oficina de Naciones Unidad contra las Droga y el Delito. (s.f.). Junta Internacional de Fiscalización de Estupefacientes (JIFE). Recuperado de https://www.unodc.org/lpobrazil/es/drogas/jife.html.

Pons, X. (2016). Materiales para la intervención social y educativa ante el consumo de drogas. San Vicente, España: Editorial Club Universitario.

Sánchez, M. \& Suelves, J. M. (2001). Asertividad y uso de sustancias en la adolescencia: resultados de un estudio transversal. Anales de Psicología, 17(1). Recuperado de http://www.redalyc.org/articulo.oa?id=16717102.

Villacís, G. (2013). Efectos físicos y psicológicos de drogas sintéticas: "una amenaza latente". Recuperado de http://repositorio.usfq.edu.ec/bitstream/23000/2299/1/107042.pdf.

\section{Derechos de autor}

Los derechos de autor de este artículo son retenidos por los autores, con los derechos de primera publicación otorgados a la revista. Este es un artículo de acceso abierto distribuido bajo los términos y condiciones de la licencia Creative Commons: Reconocimiento-NoComercial-CompartirIgual 4.0 Internacional 